\title{
Das 19. Jahrhundert
}

Sprachgeschichtliche Wurzeln des heutigen Deutsch

Herausgegeben von

Rainer Wimmer

Sonderdruck

\begin{tabular}{|l|}
$W$ \\
$D E$ \\
$G$
\end{tabular}

Walter de Gruyter · Berlin · New York 1991 


\section{INHALT}

Vorwort des Herausgebers

Eröffnungsansprache des Präsidenten des Instituts für deutsche Sprache

Willibald Steinmetz: Die schwierige Selbstbehauptung des deutschen Bürgertums: begriffsgeschichtliche Bemerkungen in sozialhistorischer Absicht

Klaus J. Mattheier: Standardsprache als Sozialsymbol. Über kommunikative Folgen gesellschaftlichen Wandels

Jürgen Wilke: Auf dem Weg zur „Großmacht”: Die Presse im 19. Jahrhundert

Forum: Sprachenpolitik gegenüber fremdsprachigen Minderheiten im 19. Jahrhundert

Ludwig M. Eichinger: Einleitung

Joachim Gessinger: Preußen 106

Heinz H. Menge: „Polen” an der Ruhr 125

Frédéric Hartweg: Elsaß 136

Claus Jürgen Hutterer: k.(u.)k. Monarchie 164

Ulrike Kiefer: Jiddisch 172

Bettina Simon: Zur Situation des Judendeutschen

Joachim Schildt: Zu einigen Entwicklungstendenzen im politischen Wortschatz der deutschen Arbeiterbewegung

Siegfried Grosse: Arbeitersprache im Ruhrgebiet 202

Isa Schikorsky: Zwischen Privatheit und Öffentlichkeit. Autobiographische Texte von Handwerkern

Angelika Linke: Zum Sprachgebrauch des Bürgertums im 19. Jahrhundert. Überlegungen zur kultursemiotischen Funktion des Sprachverhaltens 


\section{Sprachenpolitik gegenüber fremdsprachigen Minder- heiten im 19. Jahrhundert}

\section{Der historische Rahmen}

Es empfiehlt sich, allzu offenkundigen Zusammenhängen mit Skepsis zu begegnen. Wohl abgeschlossen, stimmig erscheint das Bild, das dem Leser sich vor Augen stellt, wenn von "Sprachenpolitik" gegenüber "Femdsprachen und Minderheiten" im „19. Jahrhundert" geschrieben steht. Es ist das Bild des Nationalstaats, der seine Identität nicht zuletzt aus jenem staatsgebundenen Teil der Bevölkerung nimmt, die man unter diesem Gesichtspunkt die nichtfremdsprachige, einsprachige Mehrheit nennen könnte. Die Mehrheit und die Minderheiten ergänzen sich in einem Staat, an den zumindest zwei Anforderungen zu stellen sind. Es muß sich um einen Staat handeln, in dem Mehrheitsentscheidungen und damit auch der Tatbestand, zur minderen Zahl zu gehören, vorgesehen und politisch folgenreich sind. In der Situation des 19. Jahrhunderts in Mitteleuropa kann man wohl gleich noch konkreter sagen, um einen nicht mehr aristokratischen, sondern sich formaldemokratischen Entscheidungsprozessen annähernden Staat. Die zweite Anforderung stellt eine gewisse Einschränkung des Gleichheitsprinzips dar, dessen praktische Ausübung ja im erstgenannten Punkte eingefordert wird. Sie besagt nämlich, daß die Sprache, die von einer Gruppe innerhalb des Staates gesprochen wird, nicht nur der Identifizierung als eigen- oder fremdsprachig sondern der Identitätsbildung dient.

Die Nation setzt sich nicht aus dem Willen ihrer Mitglieder zusammen, sondern deren Wille wird von ihrer Zugehörigkeit zur nationalen Totalität beherrscht [...] Und hier stimmt de Maistre mit Herder überein: 'Die Nationen haben eine Gesamtseele und eine echte innere Einheit, die sie zu dem macht, was sie sind. Diese Einheit zeigt sich vor allem durch die Sprache an'. (Finkielkraut 1989, S. 24/25)

Es ist dies zweifellos der Weg, dem Staat eine Legitimation zu geben, die über den Willensakt des freien, gleichen, brüderlichen Individuums hinausgeht und in der Lage ist, das Gottesgnadentum der aristokratischen Vorzeit in konservativerer Weise abzulösen. Es ist offenkundig, daß das egalitäre und das zuletzt angedeutete ethnische Prinzip einander widerstreiten:

Jede Gleichzeitung von Demos als Träger der politischen Souveränität mit einem spezifischen Ethnos führt im Ergebnis zu einer Unterdrückung oder Zwangsassimilation von anderen ethnischen, kulturellen, religiösen oder sozioökonomischen Bevölkerungsteilen innerhalb 
eines politischen Verbandes. So versuchte man im deutschen Reich nach 1871, die Polen in den deutschen Ostprovinzen zu germanisieren, die Elsässer und Lothringer zu verdeutschen, die Katholiken und Sozialdemokraten als national unzuverlässing - ultramontanistisch oder internationalistisch - zu diskriminieren. [...] Je nachdem, welche Eigenschaften zur Aufladung der nominalen Kategorie des Staatsbürgers verwendet werden, ergeben sich höchst unterschiedliche Diskriminierungsfälle, denn das Gleichheitsgebot zwischen den Staatsbürgern erfährt eine Brechung über zusätzliche Eigenschaften: Die ethnische Gleichheit, die religiöse Gleichbeit, die kulturelle Gleichheit oder die rassische Gleichheit. (Lepsius S. 753, nach Habermas 1990, S. 219).

Nun sind wir hier, wie man schon an den Beispielen sieht, mitten in dem Sachbereich, mit dem sich die folgenden Beiträge beschäftigen wollen, mit den Abwehrmaßnahmen gegenüber Bevölkerungsgruppen, die nach dieser Identitätsdefinition als fremd zu gelten hatten. So tritt der Nationalismus auf, der, fast per definitionem, die Minderheiten unterdrückt; nicht vergessen soll aber werden, daß es etwas anderes ist, wenn man von den Minderheiten des 19. Jahrhundert in dieser Weise spricht und wenn man von den heutigen Minderheitsverhältnissen in derselben Weise beschreibend redet. Das gilt gleichermaßen für die verwandten Begriffe wie Nationalität, Volksgruppe, Ethnie oder Minorität, die in ähnlicher Weise behandelt werden. Vielfach werden diese Verwendungen schier unauföslich vermischt, wohl als Folge des Bewußtseins, so etwas wie Minderheiten habe es wohl immer gegeben und es sei offenkundig, was das sei. Diesen Eindruck kann man etwa aus dem folgenden Beleg, einem Zitat aus dem Artikel Nationalismus, Nationalist, nationalistisch der "Brisanten Wörter” gewinnen. Dabei sei hier nicht darauf eingegangen, inwieweit ein solcher Lexikonartikel die Ambivalenzen der Verwendung lediglich wiederzuspiegeln oder auch zu reflektieren hätte:

Als entsprechend negativ eingeschätzte Kennzeichen des Nationalismus werden hervorgehoben ein übermäßig stark ausgeprägter Nationalstolz, übersteigertes Nationalgefühl oder Nationalbewußtsein sowie eine Ideologie, die sich besonders in der Überbewertung der eigenen, für höherwertig gehaltenen Nation und in der Geringschätzung oder Verachtung anderer Nationen, Völker und nationaler, rassischer oder religiöser Minderheiten ausdrückt. (Strauß/Haß/Harras 1989, S. 258)

Die Autoren dieses Artikels nehmen einen weitgehenden Konsens beim Gebrauch der Wörter aus dem Minderheiten-Feld an. Denn weder das Wort Minderheit noch Wörter wie Volk oder Nation werden in diesem Wörterbuch als erklärungsbedürftig betrachtet, offenbar auch nicht in den deutlichen Stallgeruch ihrer historischen Herkunft tragenden Verbindungen mit national, rassisch oder religiös. Demgegenüber wird in der in mehrerlei Hinsicht gewichtigen Arbeit von Emil Brix über die Um- 

hingewiesen:

Der Mangel an politischer Einigung nach nationalen Gesichtspunkten - konträr zur Situation in den westeuropäischen Sprachen - führte schließlich zu dem für das gesamte 19. Jahrhundert anerkannten 'deutschen Sprachgebrauch', der den Begriff Nation auf gemeinsam Abstammung und den dadurch gegebenen ethnischen und kulturellen $\mathrm{Zu}$ sammenhang bezog, für den Nation gleich Stammesangehörigkeit war, während für die politische Einheit der Begriff Volk gebraucht wurde. Im Gegensatz zu dieser ost- und mitteleuropäischen Theorie hatte sich dort, wo die kulturelle Einigung aufgrund des politischen Verbandes längst vollzogen war, das heißt im angelsächsischen und teilweise im romanischen Bereich, eine einheitliche Terminologie für den politischen und kulturellen Verband ausgebildet. Für beide wurde der Begriff Nation verwendet. Gemäß dieser westlichen Theorie wurde unter Nationalität die Zugehörigkeit zu einer politisch geeinten Einheit, die Staatsangehörigkeit, verstanden [...] . Daneben gab und gibt es den Begriff der Staatsnation im subjektiven Sinn. Sie umfaßt den Verband der vom Willen und Konsens zur gemeinsamen statlichen Existenz beseelten Bürger. (Brix 1982, S. 22/23)

Daß dieser Gegensatz im 19. Jahrhundert von erheblicher Bedeutung war, wird einleuchtend durch das Historikergefecht um die Position von Elsaß-Lothringen gezeigt, das nach dem Krieg von 1870/71 zwischen französischen und deutschen Historikern geführt wurde (vgl. Finkielkraut 1989 , S. $36 \mathrm{ff}$.).

\section{Mittel der Beschreibung}

Die geschilderte Ambivalenz des modernen Minderheitenbegriffs, die sich aus diesen beiden Quellen speist, spiegelt sich auch in einer gerade erschienen Publikation aus dem Institut für deutsche Sprache, in der Joachim Born und Sylvia Dickgießer einen Überblick über „Deutschsprachige Minderheiten" geben, und wegen der damit verbundenen über das rein Linguistische hinausgehenden Bedeutungsteile von deutschsprachigen Minderheiten statt von „Deutsch als Muttersprache im nicht deutschsprachigen Ausland" sprechen - was man auf jeden Fall auch schon aus ästhetischen Gründen begrüßen wird. Allerdings stehen sie dann auch vor dem Schwammcharakter des Minderheitenbegriffs; ausgehend vom Aufkommen subjektiver Zugehörigkeitskonzepte ( sprachliche Minderheit ohne Sprache"; Bewußtseinsminderheit) wird räsoniert:

Sie leine kommentarlose Verwendung des Begriffs „deutsche Minderheit"/L.E.] ermöglicht zum Beispiel dem Rezipienten die Projektion eines an subjektiven Kriterien orientierten Nationalitätsbegriffs auf Situationen, in denen kein dem unseren vergleichbares Bewußtsein von 
Volksgruppenzugehörigkeit existierte. Es sei in diesem Zusammenhang daran erinnert, daß die uns bekannte Nationalitätsproblematik ein Produkt des ausgebenden 18. und des 19. Jahrhunderts ist. Davor bestimmten Fragen der Religionszugehörigkeit, die von existentieller Bedeutung waren, das jeweilige Gruppenbewußtsein weitaus stärker als viele andere kulturelle Werte. Daher kann beispielsweise von Mitgliedern protestantischer Kollektive, die im 17. Jahrhundert unter anderem aus der Pfalz nach Nordamerika auswanderten, nicht angenommen werden, daß sie über ähnliche Kriterien der kulturellen Zuordnung verfügten wie zum Beispiel Deutschsprachige in den Sudentenländern, die aufgrund ihrer ursprünglich nach Österreich hin orientierten kulturellen Identität nach 1918 für eine Eingliederung ihres Siedlungsgebiets in den österreichischen Reststaat und aufgrund ihres (groß)deutschen Nationalbewußtseins in den dreißiger Jahren für eine Eingliederung in das nationalsozialistische deutsche Reich plädierten. Die Tatsache, daß beide Gruppen als 'deutsch' bezeichnet werden, darf nicht darüber hinwegtäuschen, daß sie sich in ihrer, eigenen kulturellen Zuordnung unterschieden. Gemeinsam ist ihnen im wesentlichen die linguistische Klassifikation ihrer Idiome als Varietäten der deutschen Sprache. (Born/Dickgießer 1989, S. 11)

Was heißt es dann aber, wenn beide Gruppen unter dem Obertitel deutschsprachige Minderheiten laufen? Doch wohl nur, daß sie mit ihrer Sprache in der staatlichen Organisationsform, innerhalb der sie heute leben, in der Minderzahl, der Unterzahl sind. Dies ist zweifellos nicht die präferierte, auch nicht die interessanteste Leseart des Begriffs Minderheit im Zusammenhang von Sprachkontakt, wichtiger ist hier das Konfliktpotential, das beim Zusammentreffen konkurrierender Sprachformen auftritt. Aus diesem Blickwinkel ist die Minderheit zuvörderst eine in irgendeiner Weise von der in einem Staatswesen herrschenden Norm abweichende Gruppe, die aufgrund ihres Abweichens ihres daraus folgenden minoritären Status, Nachteile zu gewärtigen hat, ihre eigene Identität nicht entfalten kann. ${ }^{1}$ Die klassische Minderheit ist daher die unterdrückte Minderheit, die sich zu emanzipieren wünscht: Das geschieht nun in der Phase der Entwicklung von Nationalstaaten als Tendenz, die eigene "Nation" auch in einem eigenen Staat repräsentiert zu sehen.

1 Dazu paßt etwa die Beschreibung des Begriffs "Nationalismus B" in den "Brisanten Wörtern" (S. 260), wo eine positive Bewertung dieses Konzepts für die nach staatlicher Selbständigkeit strebenden Länder der dritten Welt konstatiert wird. 


\section{Achtzehntes, neunzehntes und zwanzigstes Jahrhundert}

An dieser Stelle nun wird das 19. Jahrhundert tatsächlich interessant, allerdings eher, insofern in seinem Verlauf Organisationsformen gefunden werden, in denen die im 18. Jahrhundert angestellten Überlegungen, die besonders in der zweiten Hälfte dieses Jahrhunderts zu einer beschleunigten Umwandlung der geselschaftlichen Strukturen führten, ihren Niederschlag fanden. Diese Etappe der Beschleunigung und des Umbruchs ist von Soziologen wie Niklas Luhmann und Historikern wie Reinhart Koselleck gut festgemacht und beschrieben (vgl. z.B. Koselleck 1979; Luhmann 1980). Interessant für unser Thema ist nicht so sehr, wie dieser Übergang abläuft, sondern wie sich die Konstellation der Faktoren, die für die Interpretation der gesellschaftlichen Wirklichkeit als relevant angesehen werden, in einer geänderten Redeweise bzw. in Bedeutungsveränderungen von Wörtern niederschlägt. Dieser Prozeß eines Diskurswandels betrifft auch das Reden in den Minderheiten, tatsächlich ist das Wort als solches nach Auskunft der Wörterbücher erst seit dem beginnenden 19. Jahrhundert belegt. Wie in vielen verwandten Fällen handelt es sich um eine Lehnübersetzung aus dem Französischen, dessen kulturelle Führungsrolle hier noch einmal sichtbar wird. Die 21. Auflage des Klugeschen etymologischen Wörterbuchs beschreibt den Vorgang in der prägnantest denkbaren Weise:

Minderheit f. für Minorität, das aus frz. minorité während der Revolution zu uns gelangt und seit 1792 zu belegen ist [...] bucht Kramer 1887 Minderkeit. Campe setzt Minderheit durch [...] . Daß Notker um 1000 ahd. minnerheit für lat. minoritas gebildet hatte, wußten beide nicht. (Kluge ${ }^{21} 1975$, S. 479)

Der Hinweis auf Campes Althochdeutsch-Unkenntnis gibt Gelegenheit zu einer etwas ernsthafteren Bemerkung. Denn: Althochdeutsch oder nicht althochdeutsch, selbstverständlich wurde auch vorher das Problem „des minderen Teils" bei Entscheidungen diskutiert. Vor allem die im gesamten Zeitraum der Aufklärung mit unterschiedlicher Akzentsetzung diskutierten Konsenstheorien der Gesellschaftsentstehung brachten immer wieder die Frage nach Berechtigung der Mehrheitsentscheidung aufs Tapet, insgesamt mit dem eher harmonistischen Schluß, die Meinung der Mehrheit garantierte auch die Herrschaft der Vernunft. Das ist auch weiter nicht so erheblich, wenn in der Regierungsform keine Möglichkeiten eingebaut sind, die eine Mehrheitsentscheidung politisch bedeutsam machen. Dem absolutistischen Fürsten ist der sprachliche Unterschied allenfalls ein praktisches Hindernis.

Interessant wird das Mehrheits/Minderheitsverhältnis dann, wenn die Basis des Zusammenlebens von solchen Entscheidungen betroffen ist. 
Theoretisch wird diese Zeit eingeläutet mit den naturrechtlichen Vertragstheorien der Gesellschaftsentstehung, die im 18. Jahrhundert bei John Locke und Jean Jacques Rousseau in unterschiedlicher Akzentsetzung kulminieren (vgl. Jäger 1982, S. 1028ff.). Recht bald werden vor allem in der amerikanischen Diskussion auch die Gedanken des Minderheitenschutzes entwickelt. In Deutschland kam etwa mit Herder, der an dieser Stelle auf Condillac aufbaut, aber auch gesamteuropäisch als folgenreicher eingeschätzt werden kann, der Gedanke auf, daß Sprache und Nation unmittelbar zusammenhingen. Bei ihm verbindet sich eine letztlich antiaristokratisch wirksame Konzeption von Volk mit der Konstitution dieser Gruppenindividualität durch die gemeinsame Sprache, den Genius der Sprache. Diese Parallelsetzung von Nation und Sprache und der Nation als eigene Individualität, wie sie Wilhelm von Humboldt noch schärfer fassen wird, steht nun einerseits politisch an entscheidender Stelle, verstärken sich doch mehr und mehr die nationalstaatlichen Tendenzen, die notwendig zu Minderheitenproblemen führen, andererseits auch an einer entscheidenden Stelle des Sprachausbaus (vgl. Eichinger $1989 b)$.

Was die nationalstaatlichen Tendenzen und ihre Folgerungen für die Sprachenpolitik angeht, so mag auf die Signalwirkung der französischen Revolution und ihrer Sprachenpolitik gewiesen werden. In ihrer wohl berühmtesten Programmschrift, die der Abbé Gregoire im Jahre 1794 vorlegt, wird die Französisierung unter ausdrücklichem Verweis auf Herder und die identitätsprägende Kraft der Sprache begründet (s. Eichinger 1989a, S. 54f.). Im Verlaufe der nationalen Aufschwünge in Deutschland der antinapoleonischen Kämpfe wendet sich das natürlich gegen die Vorherrschaft auch des Französischen und fordert die gleichberechtigte Anerkennung der jeweiligen Sprachen in ihren Staatsgebieten. Gleichermaßen ist aber mit dem Ende des 18. Jahrhunderts zumindest für das Deutsche die Phase des Ausbaus zu einer in allen Domänen brauchbaren Sprachform weithin abgeschlossen. Die Ablösung vom Lateinischen ist weithin geschehen, die vom Französischen steht knapp bevor. Daraus folgt in zweierlei Hinsicht ein neues Selbstbewußtsein der Sprecher des Deutschen, das auch im nationalen Bereich nach einer Gleichstellung mit der Vorbildmacht Frankreich drängt und andererseits einen Vorsprung gegenüber anderen, sich erst im Verlaufe des 19. Jahrhunderts emanzipierenden Sprachen wie den slawischen hat. Deren nationale Identitätsfindung leidet dann schon unter weiteren Einflüssen, die die Irrelevanz der nationalen Unterschiede offenbar zugunsten eines sprachlichen Status quo betonen, nweshalb Marx 1848 den Tschechen 'Krieg bis zum Tod, Ausrottung ohne Rücksicht' ankündigte, weil sie als Minorität der industriellen und politischen Revolution und damit der Emanzipation nur 
hinderlich seien" (Bergeron/Furet/Koselleck 1969, S. 295). Das immerhin im selben Jahr, wo in der Paulskirchenverfassung den Minderheiten ein freier Gebrauch ihrer Muttersprachen zugesichert wird. Nach der 48er Revolution wächst dann das einzelstaatlich-antidemokratische Machtstreben vor allem in Preußen und Österreich so stark, daß zentralistischmonarchistische Herrschaftsmodelle fast wieder alleinherrschend werden. Nun beginnt langsam das, was man modern eine nationalistische Minderheitenpolitik nennen könnte, sie nur eine Sprachenpolitik zu nennen, ist vermutlich zu wenig.

Ein weiterer Punkt, der die sprachliche Entwicklung des 19. Jahrhunderts kennzeichnet und in direkter Beziehung zur Sprachenpolitik gesehen werden kann, ist die Durchsetzung der Einheitssprache im Pflichtschulunterricht, die ja auch im Verlaufe dieses Jahrhunderts zu einem gewissen Ende kommt. Auch hier sind sicherlich die Vorbilder einer erreichten Standardisierung der Sprachform die gesellschaftlich avancierten Staaten, vor allem Frankreich und England - kein Wunder, daß im Lauf der geschilderten Entwicklung die Tendenz zunahm, die Schule als nationales Initiationsinstrument zu nutzen. Dabei soll aber nicht vergessen werden, daß die gemeinsame Sprache nicht nur als ein Unterdrückungsmittel verstanden werden kann, vielmehr auch als Garant der Freiheit und Gleichheit angesehen werden muß.

Das gilt umso mehr, wenn man sich fragt, was die sprachlichen Folgen der Sprachenpolitik sein könnten; die jahrhundertelange Existenz hoch- und schriftsprachlicher Bildung hat hier die Verhältnisse gänzlich verändert und bei der Beurteilung der historischen Fragen ist zu bedenken, daß ja ohnehin der Schritt von der weitgehenden Oralität zur Einübung in die Schriftlichkeit voller Mühsal ist, ungeachtet der sprachlichen Verwandtschaft oder Nichtverwandtschaft.

Das Minderheiten-Thema ist, wie zu sehen war, ein Thema des 19. Jahrhunderts, in seiner ersten Hälfte konkretisieren sich Entwicklungen, die in den nationalstaatlichen Fixierungen der zweiten Jahrhunderthälfte ihre Form finden. Jedoch - zwei Punkte bleiben festzuhalten: Wenn man in der Mitte des Jahrhunderts einen solchen Umschwung sieht, so ist das auch ein Umschwung, der ein Ende macht mit den Ideen, die aus dem 18. Jahrhundert kommen, und einen Beginn setzt, dessen Folgen weit in das 20. Jahrhundert reichen. So stellt sich das 19. Jahrhundert als der Zeitraum dar, in dem die Idee der Emanzipation kollektiver Volkssubjekte, die man an ihren Worten, ihrer Sprache, erkennen sollte, eine erfolgreiche Organisationsform fand. Diese staatliche Form war zur Konfliktvermeidung dem Ausmaß der angenommenen Volkssubjekte anzupassen. Diese Angleichungsprozesse werden als der zentrale gesellschaftliche Konflikt 
verstanden, da jegliche Sprachnation ihr eigenes kohärentes und von dem anderer Nationen getrenntes Weltbild habe. Im Zusammenhang mit den vorliegenden politischen Machtverhältnissen und einem durch die Wendung des wirtschaftlichen Lebens gegebenen Modernisierungsdruck führt das in unserem Zeitraum durchgehend zu einer konsequenten Assimilierungspolitik gegenüber den stark minoritär gewordenen Minderheiten, oder von der anderen Seite gesehen, zu der Tendenz der Minderheit, sich selbst eine nationale Organisationsform zu erkämpfen; wobei das Erkämpfen mehr oder minder metaphorisch gemeint sein kann.

\section{Postnationale Minderheiten}

Nun hat das so begründete Konzept spätestens seit der Zeit des Nationalsozialismus endgültig seine Wirkung verloren. Wo denn die staatliche Identität, soweit sie existiert, sich aus einer Kombination von verschiedenen Quellen speist, spielen voluntaristische Elemente wieder eine größere Rolle. Gleichermaßen tritt mit dieser Entwicklung die Neigung zu monokausalen Erklärungen zurück, es steigt dagegen die Bedeutung des demokratisch legitimierten Systems und seiner Institutionen. Die weithin säkularisierte, ihres Glaubenscharakters entkleidete, organisatorische Hülle des westeuropäischen demokratischen Nationalstaates dient als eine normative Instanz, die uns hilft ${ }_{n}$ eine Insel im aufgewühlten Meer der Komplexität" (s. Weinrich 1973, S. 145) zu finden. Moderne gesellschaftliche Entwicklungen dienen nicht mehr der nationalen Emanzipation, die Existenz von Nationalstaaten und tragenden Staatsnationen ist vorausgesetzt, sie stehen im Zentrum der existierenden, als einheitlich und egalitär verstandenen Staatsgebilde:

Die politischen Ideologien des 19 . Jahrhunderts versprachen immerhin noch eine globale Orientierung innerhalb einer hochkomplexen, zur zweiten Natur geronnenen Gesellschaft. Heute hat ein solcher Weltbildersatz, jedenfalls in den relativ offenen Massenkulturen des Westens, keine Chance". (Habermas 1990, S. 91)

Was hier formuliert ist als der hoffnungsvolle Glaube der Aufklärung, ist ein deutlicher Niederschlag des Versuchs, historische Gebundenheit und übergreifende Geltung nationaler Identitätskonzepte zu umreißen. Für das Minderheiten-Thema wirken solche Überlegungen, wie die Skepsis gegenüber der Gültigkeit eines 19. jahrhundertlichen Minderheitenbegriffs überhaupt, wie Widerhaken in der allgemeinen Glätte des Konsens über die Interpretation minoritärer Existenz. Das hat Folgen, wenn man das Rahmenthema dieses Kongresses ernst nimmt, das ja von den Folgen des 19. Jahrhunderts für unsere heutige Zeit spricht. Die Identifikation mit den Forderungen eines Minderheitenkonzepts, das Gerechtigkeit im Rahmen nationaler Identität definiert, vermag nicht auf eine einfache 
Weise auf die Gleichheitsansprüche in modernen Gesellschaften übertragen zu werden. Dennoch ist der Geltung solcher Interpretationszüge Rechnung zu tragen: Sie definieren mit, in welchem Rahmen das Minderheitenspiel jetzt gespielt wird.

\section{Die Beiträge}

Unter diesem Gesichtspunkt beschreiben die Beiträge der Forumsveranstaltung, die hier dokumentiert wird, nicht nur verschiedene Konsequenzen aus einer historischen Deutungs- und Handlungskonzeption, sondern liefern Facetten für die Begründung normativer Erwartungen in unserem öffentlichen Leben. Als Überlegungen dazu, wie das Konzept Minderheit mit seiner historischen Entwicklung bei der Interpretation moderner $\mathrm{Ge}$ sellschaften wirksam werden kann, kann man sie auch interpretieren als Beitrag dazu, daß Mitglieder einer Kultur „zu ihren eigenen Überlieferungen eine hypothetische Einstellung einnehmen und auf deren Grundlage kulturelle Selbstrelativierungen vornehmen können" (Habermas 1990, S. 125).

So sind auch die im folgenden besprochenen Beispiele für die Sprachenpolitik gegenüber fremdsprachigen Minderheiten die Fälle, die auch im heutigen Bewußtsein am leichtesten der Selbstrelativierung dienlich sein können. Wenn man diese doppelte Funktion bedenkt, nimmt es nicht wunder, daß Preußen und später das unter seinem Einfluß stehende Deutsche Reich das Agieren der deutschen Staats- und Mehrheitsnation im Minderheitenrahmen demonstrieren. Die Politik gegenüber den Sorben, deren sich Joachim Gessinger annimmt, ist geprägt von der Existenz eines politischen und dem Bewußtsein eines kulturellen Gefälles. Ähnliches ließe sich von den Polen im Ruhrgebiet sagen, von denen Heinz M. Menge zudem zeigt, wie aus dem Blickwinkel der herrschenden Mehrheit hier verschiedene Bevölkerungsgruppen zu einer Minderheit zusammengedeutet werden, außerdem natürlich, welche Auswirkungen die wirtschaftliche Modernisierung hier hat. Zwei mit wechselnder Intensität und Aggressivität um die politische und kuiturelle Vorherrschaft streitende Nationen schildert Frédéric Hartweg im Elsaß, wo zudem die Konzepte der Geburts- und der Wahlnation aufeinandertreffen. Claus Jürgen Hutterer behandelt die Lösungsversuche für die Minderheitenprobleme in der österreichisch-ungarischen Monarchie, dem letztlich unterlegenen Widerpart Preußens in der deutschen Lösung des Nationalstaatsproblems des 19. Jahrhunderts. Wiewohl die Habsburger Monarchie von der Zusammensetzung ihrer Bevölkerung her die Chance gehabt haben sollte, den Ausgleich zwischen den Kulturen zu proben, spielt auch sie, je länger je mehr, das nationale Spiel. 
Ging es in den bisherigen Konstellationen aber immer um eine Konkurrenz von Sprachen, deren eigenständiger, nationsfähiger Status mehr oder minder außer Frage stand, ist gerade das eines der Probleme des zuletzt behandelten Falles, des Jiddischen. Auch die beiden die Lage der Sprecher dieser Sprache hier schildernden Beiträge, der von Frau Ulrike Kiefer, der auf der Tagung vorgetragen wurde, und der von Frau Bettina Simon, entscheiden sich in diesem Punkt unterschiedlich, und kommen daher auch soziolinguistisch zu unterschiedlichen Schlüssen.

Bedauerlicherweise ist diese Gegenüberstellung nicht dem Wunsch nach wissenschaftlicher Vollständigkeit oder Ausgewogenheit entsprungen. Vielmehr ist Frau Simon, die zugesagt hatte, das Referat zum Jiddischen auf der Jahrestagung zu übernehmen, während der Vorbereitungszeit zu dieser Veranstaltung an einer heimtückischen Krankheit gestorben. Es ist den Eltern von Frau Simon zu verdanken, daß hier ein Aufsatz ihrer Tochter abgedruckt werden kann. Sie haben aus den ihnen vorliegenden Vorarbeiten einen Text zusammengestellt, wie ihn Frau Simon ähnlich wohl auf der Tagung hätte vortragen wollen. Der Abdruck sei als Reverenz gegenüber der wahrlich allzufrüh aus dem Leben gerissenen Wissenschaftlerin Bettina Simon verstanden.

\section{Literatur}

Bergeron, Louis/Furet, François, Koselleck, Reinhart (1969): Das Zeitalter der europäischen Revolution (1780-1848). Frankfurt/M.

Born, Joachim/Dickgießer, Sylvia (1989): Deutschsprachige Minderheiten. Ein Überblick über den Stand der Forschung für 27 Länder. Mannheim.

Brix, Emil (1982): Die Umgangssprachen in Altösterreich zwischen Agitation und Assimilation. Wien usw.

Coulmas, Florian (1985): Sprache und Staat: Studien zur Sprachplanung und Sprachpolitik. Berlin/New York.

Eichinger, Ludwig M. (1989a): Die Dialekte und Regionalsprachen und ihr Verhältnis zur Hochsprache. Die Lage in Deutschland und Frankreich. In: Hättich, Manfred/Pfitzner, Paul Dietmar (Hrsg.): Nationalsprachen und die Europäische Gemeinschaft. München, S. 53-63.

Eichinger, Ludwig M. (1989b): Von Leuten, die nicht bis drei zählen können. Zum Weltbild der Sprachen. In: Jahrbuch Deutsch als Fremdsprache 15, S. 30-49.

Finkielkraut, Alain (1989): Die Niederlage des Denkens. Hamburg. 
Francis, E.C./Raschhofer, Hermann (1961): Minderheiten. In: Staatslexikon: Recht, Wirtschaft, Gesellschaft. Hg. v.d. Görresgesellschaft. Bd. 6. Freiburg, Sp. 715-725.

Habermas, Jürgen (1990): Die nachholende Revolution. Frankfurt/M.

Jäger, Wolfgang (1982): Mehrheit, Minderheit, Majorität, Minorität. In: Brunner, Otto/Conze, Werner/Koselleck, Reinhart (Hrsg.): Geschichtliche Grundbegriffe. Historisches Lexikon zur politisch-sozialen Sprache in Deutschland. Bd. 3 H - Me. Stuttgart, S. 1021-1062.

Kluge, Friedrich (1975): Etymologisches Wörterbuch der deutschen Sprache. 21. Auflage. Berlin/New York.

Koselleck, Reinhart (1979): Vergangene Zukunft. Zur Semantik geschichtlicher Zeiten. Frankfurt/M.

Leenhardt, Jacques/Picht, Robert (Hrsg.): Esprit/Geist. 100 Schlüsselbegriffe für Deutsche und Franzosen. München/Zürich.

Luhmann, Niklas (1980): Gesellschaftsstruktur und Semantik. Frankfurt/M.

Strauß, Gerhard/Haß, Ulrike/Harras, Gisela (1989): Brisante Wörter von Agitation bis Zeitgeist. Ein Lexikon zum öffentlichen Sprachgebrauch. Berlin/New York.

Weinrich, Harald (1973): System, Diskurs, Didaktik und die Diktatur des Sitzfleisches. In: Maciejewski, Franz (Hrsg.): Theorie der Gesellschaft oder Sozialtechnologie. Bd.1. Frankfurt/M., S. 145-161. 